\title{
Fall Detection and Classifications Based on Time-Scale Radar Signal Characteristics
}

\author{
Ajay Gadde, Moeness G. Amin, Yimin D. Zhang*, Fauzia Ahmad \\ Center for Advanced Communications \\ Villanova University, Villanova, PA 19085
}

\begin{abstract}
Unattended catastrophic falls result in risk to the lives of elderly. There are growing efforts and rising interest in detecting falls of the aging population, especially those living alone. Radar serves as an effective non-intrusive sensor for detecting human activities. For radar to be effective, it is important to achieve low false alarms, i.e., the system can reliably differentiate between a fall and other human activities. In this paper, we discuss the time-scale based signal analysis of the radar returns from a human target. Reliable features are extracted from the scalogram and are used for fall classifications. The classification results and the advantages of using a wavelet transform are discussed.
\end{abstract}

Keywords: Fall detection, assisted living, Doppler signature, wavelet transform, non-stationary signal.

\section{INTRODUCTION}

One of the major public health problems is elderly falls. Prompt assistance after a fall can reduce complications and save lives. Therefore, it is very important to detect a fall immediately as it happens and mobilize first responders for proper care and attendance to possible injury. In recent years, many fall detection systems have been proposed in the literature. These can be categorized into two main types of fall monitoring devices, namely, wearable and non-wearable. The simplest wearable device is a "push-button", which can be manually activated in case of a fall. Accelerometer-based wearable devices detect falls by measuring the applied acceleration along the elevation dimension. ${ }^{1}$ The wearable devices are inexpensive but have two main drawbacks. First, these devices cannot be activated in case of a loss of consciousness after a fall. Second, due to memory and suitability issues, the elderly may not be wearing them at all times. $^{2}$ Among the non-wearable devices, floor vibration sensors and microphone arrays have been proposed. ${ }^{3}$

Radar is an excellent modality due to its capability of detecting human motions. The general concept of radar-based system is to transmit an electromagnetic (EM) wave over a certain frequency range and analyze the radar returns. Changes in the properties of the returned signal relative to the transmitted signal depend on the target motion characteristics. In particular, the transmitted and received frequencies differ by a carrier shift, known as Doppler frequency shift or Doppler effect. The Doppler frequency shift depends on the velocity of a moving target. In addition, the motions of arms and legs introduce additional frequency modulations on the returned radar signal, which generate sidebands about the target's Doppler frequency, called the micro-Doppler effect. ${ }^{4}$ A human in motion reflects radar signals with Doppler modulations that reveal information about the motion dynamics. As such, the velocity of moving objects can be estimated from the measured Doppler frequency signature of the radar returns.

The Doppler signatures measured with biometric radars have received significant interest over the past few years. ${ }^{5-8}$ Radar sensors can provide valuable information about human body motion and cross-motor activities, and can be operated at all times. ${ }^{5}$ Gait characterization using various machine learning algorithms has proven to be very effective in terms of rendering high classification rates. ${ }^{6,7}$ In Ref. [6], six features were chosen from the short-time Fourier transform (STFT) of the radar signal to represent the micro-Doppler signatures; a support vector machine (SVM) was then used as a classifier. In Ref. [7], using mel-frequency cepstral coefficients (MFCC), the Doppler signatures of human activities are extracted and, based on these features, two different machine learning algorithms, i.e., SVM and k-nearest neighbor, are employed to detect falls. Hidden Markov model (HMM) based machine learning approach has also been applied for recognizing human actions. ${ }^{8,9}$

*Contact information: yimin.zhang@villanova.edu 
This paper considers the classification of two human activities, namely, fall and sit/stand. The raw data, collected from a continuous-wave (CW) Doppler radar, was processed to distinguish between the two motion categories of a normal sit or stand and a general fall. Wavelet transform serves as an analysis tool to analyze the non-stationary signal's time-scale characteristics. Wavelet transform and its squared magnitude, referred to as the scalogram, are applied to generate important motion attributes. Specific features are first extracted from the scalogram and then used by the Mahalanobis distance classifier to map the attribute set to the appropriate motion class.

The remainder of the paper is organized as follows. In Section 2, the signal model is presented. Continuous wavelet transform (CWT), the features extracted from the scalogram, and the Mahalanobis distance classifier are described in Section 3. Section 4 presents experimental results along with appropriate observations. Section 5 contains the conclusion.

\section{SIGNAL MODEL}

A monostatic $\mathrm{CW}$ radar transmits a sinusoidal signal, expressed as $s(t)=\exp \left(j 2 \pi f_{c} t\right)$, where $f_{c}$ is the carrier frequency. Consider a point target which is located at a distance of $R_{0}$ from the radar at time $t=0$, and moves with a velocity of $v(t)$ in a direction forming an angle of $\theta$ with the radar line-of-sight. As such, the distance between the radar and the target at time instant $t$ is

$$
R(t)=R_{0}+\int_{0}^{t} v(u) \cos (\theta) d u .
$$

The received radar signal can be expressed as

$$
x_{a}(t)=\rho \exp \left[j 2 \pi f_{c}\left(t-\frac{2 R(t)}{c}\right)\right],
$$

where $\rho$ is the target reflection coefficient and $c$ is the velocity of the EM wave propagation. The Doppler frequency corresponding to $x_{a}(t)$ is

$$
f_{D}(t)=2 v(t) \cos (\theta) / \lambda_{c}
$$

where $\lambda_{c}=c / f_{c}$ is the wavelength.

For a spatially extended target, such as a human body, the radar return is the integration over the target region $\Omega$, given by

$$
x(t)=\int_{\Omega} x_{a}(t) d a .
$$

In this case, the Doppler signature is the superposition of all component Doppler frequencies. Torso or gait motions generally generate time varying Doppler frequencies, and their exact signatures depend on the target shape and motion patterns.

\section{FALL DETECTION ALGORITHM}

Wavelet transform is considered as a powerful tool in the analysis of non-stationary signals. Like the STFT, the wavelet transform uses the inner products to measure the similarity between a signal and an analyzing function. In STFT, the analyzing functions are windowed complex exponentials, and the STFT coefficients represent the projection between the windowed signal and a sinusoid in an interval of a specified length. In the wavelet transform, the analyzing function is a wavelet.

Compared to the STFT, which uses a fixed window function to capture the local frequency components, the wavelet transform exploits multi-resolution windows to achieve both coarse and high frequency resolutions for slowly and rapidly time-varying signal components, respectively. According to the uncertainty principle, ${ }^{10}$ the product of the timedomain resolution and the frequency-domain resolution is lower bounded. That is, we cannot achieve a high resolution in both the time and frequency domains at the same time. Therefore, although STFT can observe the time-varying frequency signatures, the question always arises with regard to the optimum window length for the given data to provide the best tradeoff between spectral and temporal resolutions. 
The wavelet transform, on the other hand, implements the multi-resolution concept by changing the position and scaling of the mother wavelet and thereby captures short duration, high frequency components and long duration, low frequency components. ${ }^{11}$ In the underlying application, the wavelet transform is considered particularly useful in capturing the high Doppler frequency components of the fall while protecting the low-frequency components in the data. As the wavelet transform provides the frequency of the signals and the time associated to those frequencies, it has applications in numerous fields, such as signal processing of accelerations for gait analysis, fault detection, design of low power pacemakers, and also in ultra-wideband (UWB) wireless communications.

\subsection{Continuous Wavelet Transform}

Since basis orthogonality is not required neither is the inverse transform employed in the processing at hand, we use the CWT, rather than the discrete wavelet transform (DWT), for the processing of a discrete signal. In so doing, we can incorporate various scales and time shifts over the given data record. In essence, unlike the DWT where dyadic representation is adopted, the CWT can operate at every scale, ranging from that of the original signal up to some maximum scale which is determined by computations and analysis tradeoffs. Also, the analyzing wavelet is shifted sample by sample over the full domain of the analyzed function. The CWT compares the signal to a shifted and compressed or expanded version of a wavelet. Expansion and compression of a wavelet function, which correspond to the physical notion of scale, are collectively referred to as dilation or scaling. By comparing the signal to the wavelet at various scales and positions, one obtains the coefficients as a function of these two variables.

Mathematically, the CWT of a function $f(t)$ is presented for a scale parameter, $a>0$, and position parameter, $b$, as,

$$
c(a, b ; f(t), \Psi(t))=\int_{-\infty}^{\infty} f(t) \frac{1}{\sqrt{a}} \psi^{*}\left(\frac{t-b}{a}\right) d t,
$$

where $\psi(t)$ is the mother wavelet, $*$ denotes the complex conjugate. Note that the CWT coefficients are affected not only by the values of scale and position, but also by the choice of the wavelet. In this paper, the Morlet wavelet is used as the analyzing function. ${ }^{12}$ Since the Morlet wavelet is composed of a sinusoid multiplied by a Gaussian window, which forms the typical characteristics of a radar signal, all features of the signal are effectively captured. Moreover, it has good local performance in both time and frequency domains, despite its simplicity and computational convenience. The mother wavelet function used in our work is expressed as

$$
\psi(t)=e^{-\frac{t^{2}}{2}} \cos (50 t)
$$

and is plotted in Figure 1.

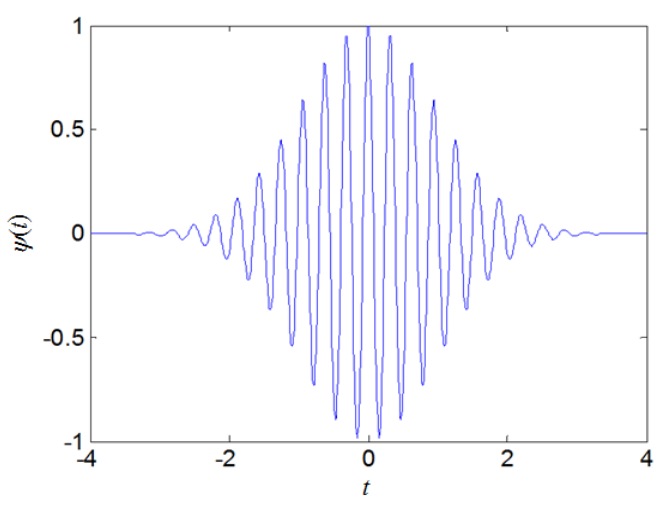

Figure 1: Morlet Wavelet.

\subsection{Wavelet Features for Classification}

To distinguish the radar signal corresponding to a fall and a sit/stand, a classifier is constructed based on the Mahalanobis distance between the features of the event under test and those corresponding to the trained fall and nonfall classes. Toward this purpose, multiple features are extracted from the signal scalogram. Only the features observed over the time period with sufficient signal power in the frequency bands of interest are passed to the classifier for fall detection. ${ }^{13}$ 
Among many possible signal attributes in the wavelet domain, we have found three features which are important and relevant to the underlying classification problem: (a) the lowest scale at which the coefficient has a significant value; (b) the ratio of the power presented from scales 1 to 200 to power presented from scales 201 to 300 ; and (c) the rate of change of scale from 300 to lowest scale. The choice of the considered scale ranges will become evident in Section 4. These features are detailed below.

(1) Lowest scale or highest frequency component. The lowest scale corresponds to the highest frequency component in the activity. This clearly helps in distinguishing between low and high velocity motions. The noise effect is mitigated by setting the scalogram coefficients below a certain threshold level, $\eta_{P}$, to zero. This threshold is determined based on the mean $\left(\mu_{P}\right)$ and standard deviation $\left(\sqrt{\sigma_{P}}\right)$ of the scalogram coefficients over the no activity region, in a spirit similar to the concept of constant false alarm rate in radar surveillance. ${ }^{14}$ The noise threshold is given by

$$
\eta_{P}=\mu_{P}+N \sqrt{\sigma_{P}}
$$

where the value of $N$ trades off between the signal preservation and noise rejection. In this paper we use $N=1.5$.

(2) Ratio of the energies. Only the ranges from 1 to 200 and 201 to 300 are selected because it is easy to distinguish sit/stand and a fall using these ranges. A typical sit/stand has significant power present above the scale of 200 , whereas a fall has significant power present even when the scale is below 100. The ratio of power from scale range of 1 to 200 to scale range of 201 to 300 is, therefore, used as a key feature for the application at hand.

(3) Rate of change of scale. The rate at which the scale changes from the 300th to the lowest scale having significant coefficient value represents how fast the Doppler frequencies vary with time.

\subsection{Mahalanobis Distance}

The Mahalanobis distance is a descriptive statistic, which is defined as the unitless measure of the distance between two points in the space defined by two or more correlated variables. ${ }^{15}$ The Mahalanobis distance is widely used in cluster analysis and classification techniques. To this end, it is used to measure the similarity between an unknown set and a known set. The unknown set is classified as the known set that has the smallest Mahalanobis distance.

Denote the mean vector of class $q$ as $\mathbf{m}^{(q)}$ and the corresponding covariance matrix as $\mathbf{C}^{(q)}$, where $q$ implies either fall or non-fall. Then, for a feature vector $\mathbf{x}$ of the event under test, the Mahalanobis distance $D^{(q)}$ between $\mathbf{x}$ and the class $q$ is expressed as

$$
D^{(q)}=\sqrt{\left(\mathbf{x}-\mathbf{m}^{(q)}\right)^{T}\left[\mathbf{C}^{(q)}\right]^{-1}\left(\mathbf{x}-\mathbf{m}^{(q)}\right)},
$$

where (.) ${ }^{T}$ denotes transpose. Note that the Mahalanobis distance differs from the Euclidean distance in the sense that it takes into account the correlations of the data set and is scale-invariant.

The computation of the features and the corresponding Mahalanobis distances is summarized below:

1) Calculate the CWT of the raw data and then segment the results into short intervals, where the actual movement takes place;

2) Generate the scalogram. That is, calculate the power of each CWT coefficient by taking the magnitude square of each coefficient;

3) Determine the mean $\mathbf{m}^{(q)}$ of the features of the training sets of each class $q$ and the corresponding covariance matrix as

$$
\mathbf{m}^{(q)}=\frac{1}{N_{q}} \sum_{i=1}^{N_{q}} \mathbf{x}_{i}^{(q)} ; \mathbf{C}^{(q)}=\frac{1}{N_{q}} \sum_{i=1}^{N_{q}}\left(\mathbf{x}_{i}^{(q)}-\mathbf{m}^{(q)}\right)\left(\mathbf{x}_{i}^{(q)}-\mathbf{m}^{(q)}\right)^{T}
$$

where $N_{q}$ is the number of training set samples for class $q$, and $\mathbf{x}_{i}^{(q)}$ is the feature vector of the $i$ th sample of the training set for class $q$.

4) Compute the Mahalanobis distance between the test feature vector $\mathbf{x}$ and the class $q$ using equation (8).

5) Assign the test vector $\mathbf{x}$ to the class with the smallest Mahalanobis distance. 


\section{EXPERIMENTAL RESULTS}

To verify the effectiveness of the proposed method, experiments were performed at the Radar Imaging Lab, Center for Advanced Communications, Villanova University. Figure 2 depicts the experiment setting. The radar operates at a carrier frequency of $8 \mathrm{GHz}$ with an antenna whose feed point is 40 inches high from the floor, and the sampling frequency is $1 \mathrm{kHz}$. The antenna is installed to transmit and receive vertically polarized signals. The test subject is approximately 9 feet away from the radar.

The experiments consist of two human subjects, who individually undergo sit, stand, and fall backward relative to the radar line-of-sight. The first experiment consists of a single human subject going through a series of sitting in a chair and standing up motions over an interval of 20 seconds. The sit and stand motions are performed in a relatively fast manner so that the Doppler frequencies are closer to those of the falls for more challenging classifications. The second experiment entails the fall of a single human followed by the motion of getting back up over a time interval of 20 seconds. Each of the two test subjects were asked to repeat both experiments five times so that variations could be monitored for all the activities. As such, the recorded data consists of ten sets per experiment. Prior to taking the wavelet transform, the clutter from the environment was suppressed by subtracting empty scene measurements from the target scene data.

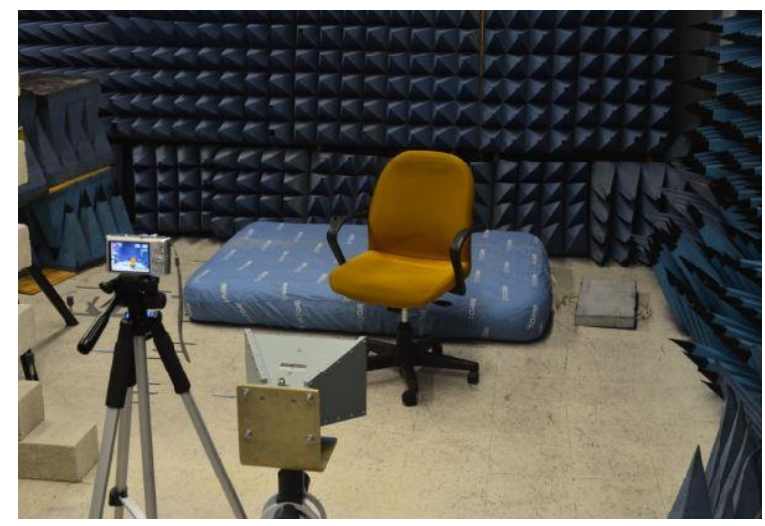

Figure 2: Experiment Setting.

The CWT of the background subtracted data corresponding to the various test cases was computed. Figures 3(a) and 3(b) show the scalograms of a fall and a sitting motion, respectively. To facilitate feature extraction, the scalograms are converted to binary images by applying threshold of noise floor, as discussed earlier. The resulting binary images are cleaned by removing those coefficients which are not representing the signal of interest. Figures 3(c) and 3(d) show the binary images corresponding to Figures 3(a) and 3(b), respectively, whereas Figures 3(e) and 3(f) depict the respective results after image cleaning. Comparing the binary images before and after cleaning, we observe that the latter provide an enhanced framework for extracting the features for distinguishing falls from sitting.

In Figure 3, the lowest scale (i.e., the highest frequency) component of a fall and sit exhibits a noticeable difference. The ratio of the power from scales 100 to 200 and 201 to 300 in the fall case is expected to be much higher because of the presence of energy components in the scale range where no activity exists for a sit case. The rate of change of scale also is expected to be much higher for a fall than a sit.

Since the CWT does not distinguish between positive and negative frequencies, sit and stand are collectively considered as one class, whereas fall is considered as another class. The classification results obtained using the Mahalanobis distance as a classifier is presented in Table 1. Two sets are taken from each class, namely, sit/stand and fall and treated as the test cases. The remaining eight data sets for each class were used as training data. The feature vector corresponding to each test set is projected onto the sit/stand and fall classes and the Mahalanobis distance to each class is determined. A small Mahalanobis distance represents a high similarity between the test set and the corresponding class. From the results in Table 1, we observe that the classification accuracy is 100\% for the sit/stand and fall classes for the data being analyzed. 


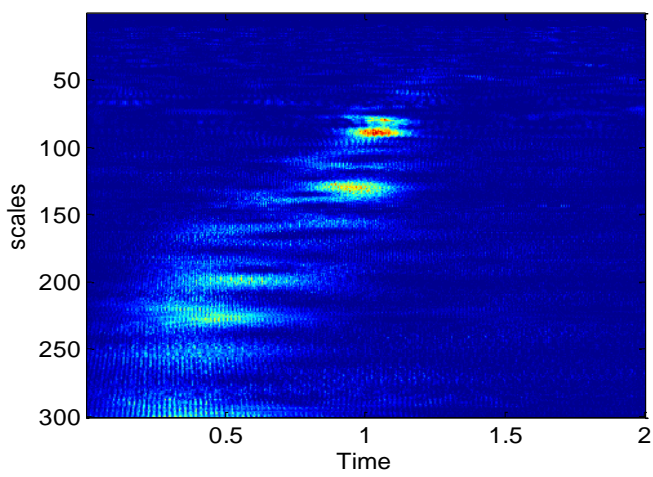

(a) Wavelet transform of a Fall

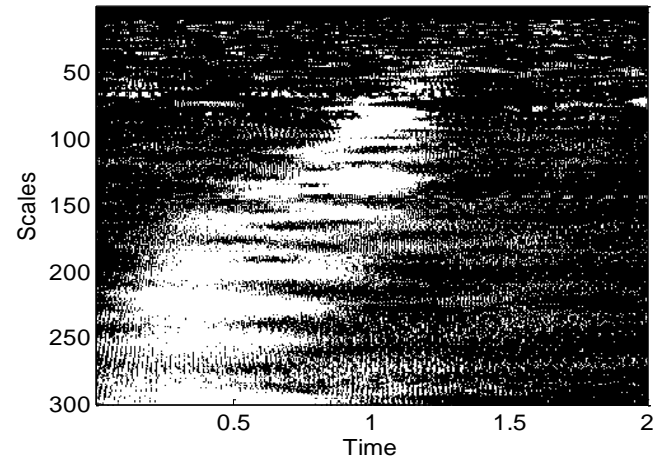

(c) Binary image of the fall scalogram

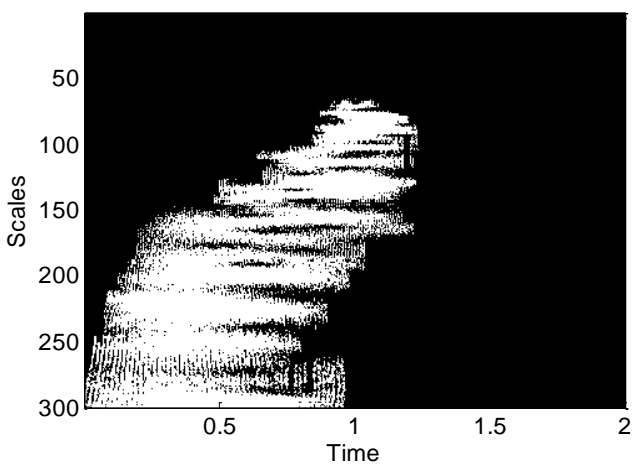

(e) Cleaned binary image of the fall

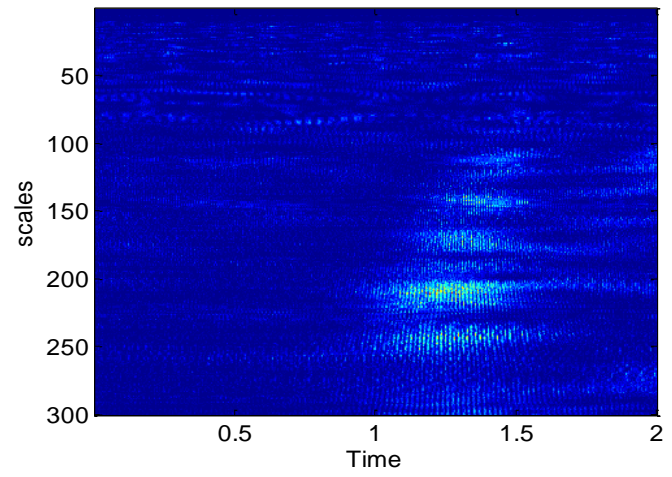

(b) Wavelet transform of a sit

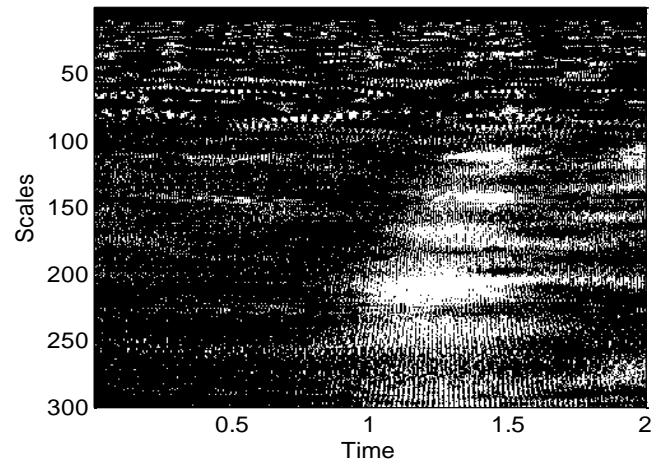

(d) Binary image of the sit scalogram

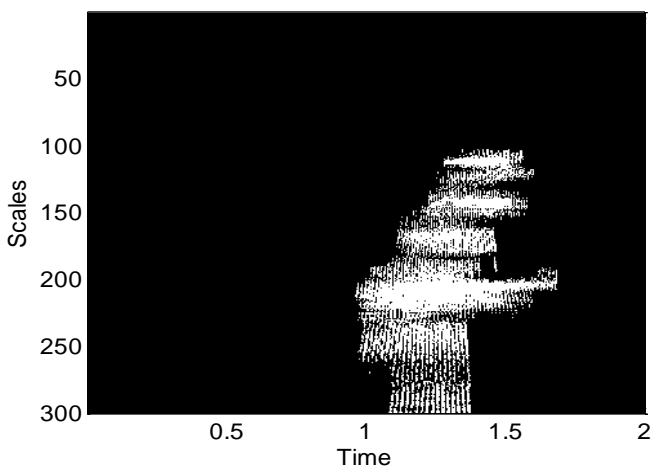

(f) Cleaned binary image of the sit

Figure 3: Scalogram and results of morphological processing.

Table 1: Confusion matrix of the classification results using CWT

\begin{tabular}{|c|c|c|}
\hline \multirow{2}{*}{$\begin{array}{c}\text { Actual } \\
\text { Class }\end{array}$} & \multicolumn{2}{|c|}{ Classified Class } \\
\cline { 2 - 3 } & Sit/Stand & Fall \\
\hline Sit/Stand & 10 & 0 \\
\hline Fall & 0 & 10 \\
\hline
\end{tabular}


To make a comparison between the scalogram-based approach with that based on the spectrogram, we first convert the cleaned scalograms of fall and sit, as depicted in Figures 3(e) and 3(f), to the time-frequency domain, which are plotted in Figures 4(a) and 4(b), respectively. The binary image of the spectrograms of the same fall and sit are plotted in Figures 4(c) and 4(d), respectively, where the Hamming window of size 255 is applied to obtain each spectrogram. From the figures, we observe that the peaks obtained from the scalogram and spectrogram are similar. The same features that are extracted from the scalogram are also obtained from the spectrogram and used to perform classification. The corresponding results using STFT are presented in Table 2, which also provides 100\% classification accuracy. However, when the window size is reduced to 127 , the STFT based classifier exhibits missed detections and false alarms, as indicated in Table 3. This example highlights the shortcoming of the STFT based approach. A suitable window size has to be specified in the STFT to obtain correct classification results. An inappropriate window size will affect the classification rate as shown in Table 3. The scalogram-based approach overcomes the limitations of the STFT based approach, thereby providing enhanced classifications. In addition, it also provides smoother distribution after the morphological processing.

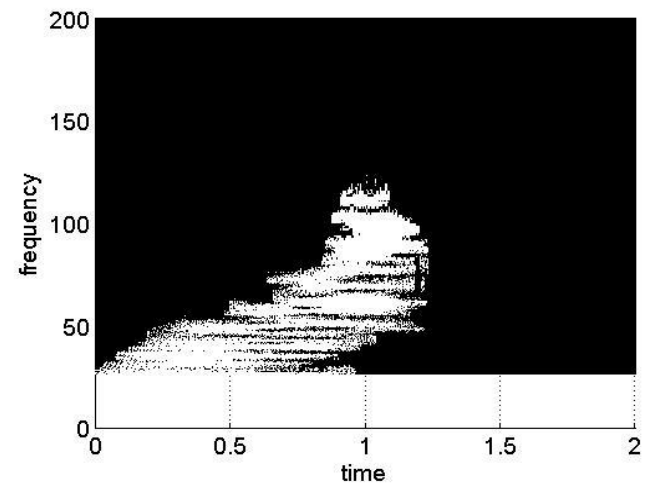

(a) Converted scalogram of the fall from Figure 3(e)

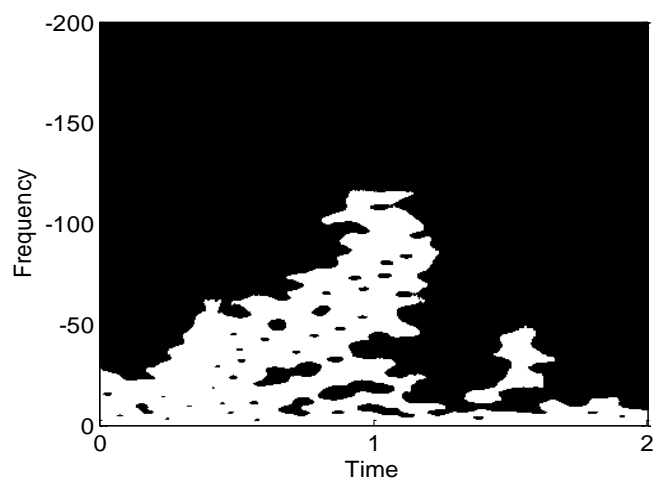

(c) Binary image of the spectrogram of the fall

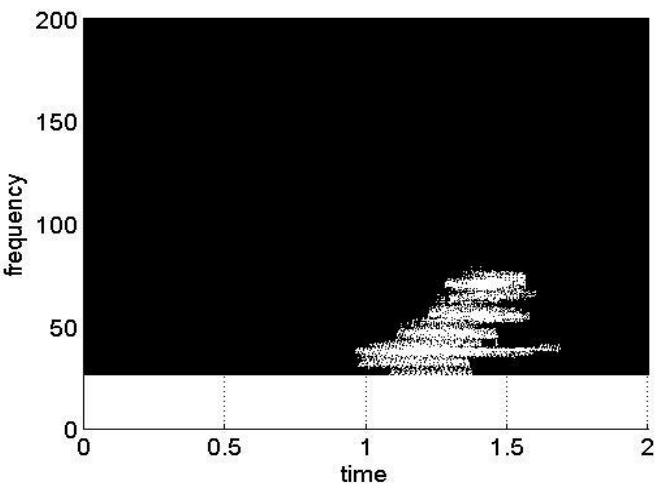

(b) Converted scalogram of the Sit from Figure 3(f)

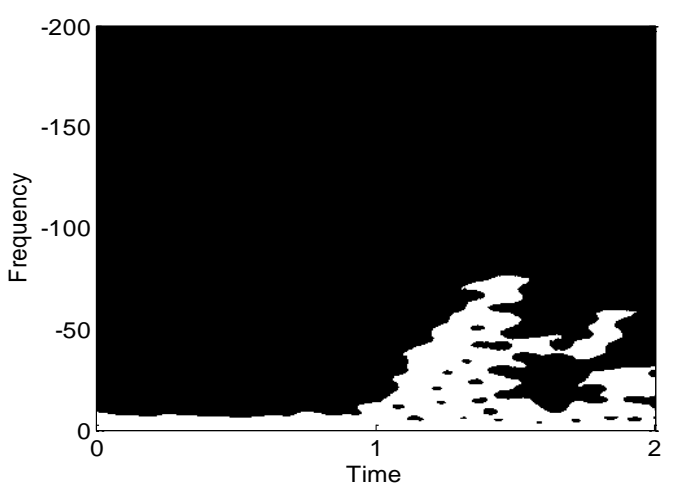

(d) Binary image of the spectrogram of the sit

Figure 4. Comparison with spectrograms

Table 2: Confusion matrix of the classification results using STFT with a window size of 255

\begin{tabular}{|c|c|c|}
\hline \multirow{2}{*}{$\begin{array}{c}\text { Actual } \\
\text { Class }\end{array}$} & \multicolumn{2}{|c|}{ Classified Class } \\
\cline { 2 - 3 } & Sit/Stand & Fall \\
\hline Sit/Stand & 10 & 0 \\
\hline Fall & 0 & 10 \\
\hline
\end{tabular}


Table 3: Confusion matrix of the classification results using STFT with a window size of 127

\begin{tabular}{|c|c|c|}
\hline \multirow{2}{*}{$\begin{array}{c}\text { Actual } \\
\text { Class }\end{array}$} & \multicolumn{2}{|c|}{ Classified Class } \\
\cline { 2 - 3 } & Sit/Stand & Fall \\
\hline Sit/Stand & 8 & 2 \\
\hline Fall & 1 & 9 \\
\hline
\end{tabular}

\section{CONCLUSION}

In this paper, the wavelet transform was used as a tool for the analysis of non-stationary radar signals that lead to the detection of typical human falls. A feature extraction technique was used as basis for classification. Three features were extracted from a scalogram obtained through the continuous wavelet transform. The extracted features were taken into a feature vector and classification was performed based on the Mahalanobis distance metric. Experimental results show a $100 \%$ classification rate between the fall and the sit/stand activities. This demonstrates that the features taken into consideration have the capability of robust classification. Since the scalogram provides a good tradeoff between the low frequency and high frequency components, classification using wavelet transform provides more accurate results as compared to the spectrogram-based counterparts.

\section{ACKNOWLEDGMENTS}

This work was supported in part by the Qatar National Research Fund under NPRP Grant \#6-680-2-282.

\section{REFERENCES}

[1] D. Giansanti, G. Maccioni and V. Macellari, "The development and test of a device for the reconstruction of 3D position and orientation by means of a kinematic sensor assembly with rate gyroscopes and accelerometers," IEEE Transactions on Biomedical Engineering, vol. 52, no. 7, pp. 1271-1277, July 2005.

[2] N. Noury, A. Fleury, P. Rumeau, et al., "Fall detection principles and methods", in Proceedings of IEEE International Conference of the Engineering in Medicine and Biology Society, Lyon, France, Aug. 2007.

[3] Y. Li, Z. Zeng, M. Popescu, and D. Ho, "Acoustic fall detection using a circular microphone array," in Proceedings of IEEE International Conference of the Engineering in Medicine and Biology Society, Buenos Aires, Argentina, Aug. 2010.

[4] V. C. Chen, "Analysis of micro-Doppler signatures," IEE Proceedings - Radar, Sonar and Navigation, vol. 150, no. 4, Aug. 2003.

[5] M. G. Amin (ed.), Through-the-Wall Radar Imaging. CRC Press, 2010.

[6] Y. Kim and H. Ling, "Human activity classification based on micro-Doppler signatures using a support vector machine," IEEE Transactions on Geoscience and Remote Sensing, vol. 47, no. 5, May 2009.

[7] L. Liu, M. Popescu, M. Skubic, M. Rantz, T. Yardibi, and P. Cuddihy, "Automatic fall detection based on Doppler radar motion signature," in Proceedings of International Conference on Pervasive Computing Technologies for Healthcare, Dublin, Ireland, May 2011.

[8] M. Wu, X. Dai, Y. D. Zhang, B. Davidson, M. Amin, and J. Zhang, "Fall detection based on sequential modeling of radar signal time-frequency features," in Proceedings of IEEE International Conference on Healthcare Informatics, Philadelphia, PA, Sept. 2013.

[9] J. Yamato, J. Ohya, and K. Ishii, "Recognizing human action in time-sequential images using hidden Markov model," in Proceeding of IEEE Computer Society Conference on Computer Vision and Pattern Recognition, June 1992. 
[10] W. J. Williams, M. L. Brown, and A. O. Hero, "Uncertainty, information and time-frequency distributions," in Proceedings of SPIE, vol. 1566, Dec. 1991.

[11] S. Qian, Introduction to Time-Frequency and Wavelet Transforms. Prentice Hall, 2001.

[12] W. Jun, "Seismic edge-preserving smoothing based on Morlet wavelet transform," in Proceedings of the IET International Conference on Wireless Mobile and Multimedia networks, Beijing, China, Sept. 2010.

[13] L. Ramirez Rivera, E. Ulmer, Y. D. Zhang, W. Tao, and M. G. Amin, "Radar-based fall detection exploiting timefrequency features," in Proceedings of IEEE China Summit and International Conference on Signal and Information Processing, Xi'an, China, July 2014.

[14] M. Skolnik, Introduction to Radar Systems, Third Edition. McGraw-Hill, 2002.

[15] S. Theodoridis and K. Koutroumbas, Pattern Recognition, Third Edition. Academic Press, 2006. 